\title{
Effectiveness of self- or partner-administration of an extended- release aqueous-gel formulation of lanreotide in lanreotide-naïve patients with acromegaly
}

\author{
Roberto Salvatori $\cdot$ Lisa B. Nachtigall • David M. Cook • \\ Vivien Bonert • Mark E. Molitch • Sandra Blethen • \\ Stephen Chang • The SALSA Study Group
}

Published online: 8 November 2009

(C) The Author(s) 2009. This article is published with open access at Springerlink.com

\begin{abstract}
Surgical resection is often not curative in patients with acromegaly and long-acting somatostatin analogues (lanreotide or octreotide) are often needed. This study assessed the efficacy and safety of self- or partner-administration of lanreotide in patients with acromegaly. This was a six-month, single-arm, open-label study conducted at 13 endocrinology clinics. Fifty-nine patients received deep subcutaneous lanreotide injections every 28 days. Twelve patients started on $120 \mathrm{mg}$ lanreotide and forty-seven started on $90 \mathrm{mg}$ lanreotide. At week 16, the dose was adjusted to 60,90 or $120 \mathrm{mg}$ based on insulin-like growth factor-1
\end{abstract}

See the Appendix for the full list of study investigators and coordinators of the SALSA Study Group.

R. Salvatori $(\bowtie)$

Division of Endocrinology, Johns Hopkins University School of Medicine, 1830 East Monument street \#333, Baltimore, MD 21287, USA

e-mail: salvator@jhmi.edu

L. B. Nachtigall

Massachusetts General Hospital, Boston, MA, USA

D. M. Cook

Oregon Health \& Science University, Portland, OR, USA

V. Bonert

Cedars-Sinai Medical Center, Los Angeles, CA, USA

V. Bonert

David Geffen School of Medicine at University of California, Los Angeles, Los Angeles, CA, USA

M. E. Molitch

Northwestern University, Chicago, IL, USA

S. Blethen · S. Chang

Tercica, Inc. a subsidiary of the Ipsen Group,

Brisbane, CA, USA
(IGF-1) levels at week 12. Fifty-nine patients with acromegaly either switched from long-acting octreotide (switch; $n=33$ ) or were somatostatin analogue treatment-naïve or not currently taking long-acting octreotide ("other"; $n=26$ ). The key endpoints included the percentage of patients/partners able to self- or partner-inject lanreotide and those with normal IGF-1 or growth hormone (GH) levels at week $24 /$ early termination. $100 \%$ of patients/partners correctly self- $(n=41)$ or partner-injected $(n=18)$ lanreotide by week 4 . By week 24/early termination, IGF-1 levels were controlled in $93.7 \%$ of switch and $46.2 \%$ of "other" patients, while GH levels were controlled in $76.9 \%$ and $39.1 \%$ of patients, respectively. Both IGF-1 and GH were controlled in $73.1 \%$ of switch and $30.4 \%$ of "other" patients. Most switch patients $(81 \%)$ reported they preferred lanreotide over long-acting octreotide for future use $(P=0.0001)$. Self- or partner-administration of lanreotide is generally well tolerated and associated with IGF-1 and GH control in many lanreotide-naïve patients with acromegaly.

Keywords Acromegaly - Self-administration · Somatostatin analogue $\cdot$ Lanreotide $\cdot$ Octreotide

\section{Introduction}

Most patients with acromegaly present with pituitary macroadenomas and are, therefore, often not cured by surgical resection even if the surgery is performed by an experienced neurosurgeon [1]. Patients with persistent growth hormone $(\mathrm{GH})$ excess after surgery require further treatment to reduce serum $\mathrm{GH}$ and insulin-like growth factor-1 (IGF-1) and to attain normal life expectancy [2]. Such treatment may include radiation or pharmacological therapy. In many instances both treatments may be required 
because radiation effects are often delayed by years [3]. Furthermore, some studies suggest that primary medical therapy with long-acting somatostatin analogues (SSAs) may be appropriate for patients who are at high surgical risk [4] or prior to surgery as this may improve the surgical cure rate [5]. SSAs, such as lanreotide (Somatuline ${ }^{\circledR}$ Depot; Ipsen Pharma Biotech, Signes, France) and longacting octreotide (octreotide acetate for injectable suspension, LAR; Sandostatin LAR $^{\circledR}$ Depot; Novatris, East Hanover, NJ), inhibit both GH secretion and tumor growth and are the mainstay of pharmacological therapies for acromegaly $[6,7]$.

Lanreotide comes in a long-acting aqueous-gel formulation that is administered via deep subcutaneous injection every 28 days and provides consistent drug release [8]. It is provided in a ready-to-use pre-filled syringe, which obviates the need for drug reconstitution prior to administration. The product is volume dependent and not concentration dependent, with volumes ranging from $0.3-0.5 \mathrm{cc}$ for delivery of the 60,90 and $120 \mathrm{mg}$ doses. A previous study reported that patients with acromegaly receiving a constant dose of lanreotide for $\geq 4$ months before screening were able to successfully self- or partner-administer lanreotide while maintaining GH and IGF-1 control [9]. We report the efficacy and safety of self- or partner-administration of lanreotide in patients with acromegaly who were lanreotide-naïve and who switched from long-acting octreotide LAR or who were SSA treatment-naïve or not currently on octreotide.

\section{Patients and methods}

Study design

This was a 6-month, single-arm, open-label, multicenter study evaluating the efficacy and safety of self- or partneradministration of lanreotide in patients with acromegaly.
Patients either switched directly from octreotide LAR (switch patients) or were SSA treatment naïve or not currently on octreotide ("other" patients; they could have had octreotide $>4$ months prior to enrollment). Patients receiving dopamine agonist (DA) treatment before the study were maintained on the same DA dose.

After screening, patients were evaluated at baseline (week 0) and at weeks 4, 12 and 24. There was an additional 30-day safety follow-up after the last lanreotide injection at week 24. Patients received deep subcutaneous injections of lanreotide every 28 days. Switch patients were started either on 90 or $120 \mathrm{mg}$ lanreotide, depending on their prior treatment regimen (e.g., typically $90 \mathrm{mg}$ if they were on $20 \mathrm{mg}$ octreotide LAR and $120 \mathrm{mg}$ if they were on $30 \mathrm{mg}$ octreotide LAR). All "other" patients were started on $90 \mathrm{mg}$ lanreotide except for one patient, who was on a DA and had previously been on $30 \mathrm{mg}$ octreotide LAR $>4$ months prior to enrollment, who started on $120 \mathrm{mg}$ lanreotide. The dose was adjusted to 60,90 or $120 \mathrm{mg}$ at week 16 based on the patient's symptoms and IGF-1 levels at week 12. A schematic illustration of the study design is shown in Fig. 1.

Patients were given the choice to self- or partner-inject lanreotide. A "partner" was defined as any person whom the patient trusted to administer the injection. The local health care professional demonstrated the injection technique at week 0 to either the patient or the partner, but the patient or partner administered all the injections.

Health care professionals completed a questionnaire assessing the competency of each patient/partner at weeks $0,4,8$ (if needed) and 24. The competency questionnaire included questions such as whether or not the patient/ partner followed the instructions given, administered the injection correctly, administered the complete dose, inserted the needle correctly and inserted the needle to the appropriate depth. Patients completed a questionnaire inquiring about acromegaly symptoms at weeks 0,12 , and 24 and an injection diary after each injection. A total score
Fig. 1 Schematic representation of the study design

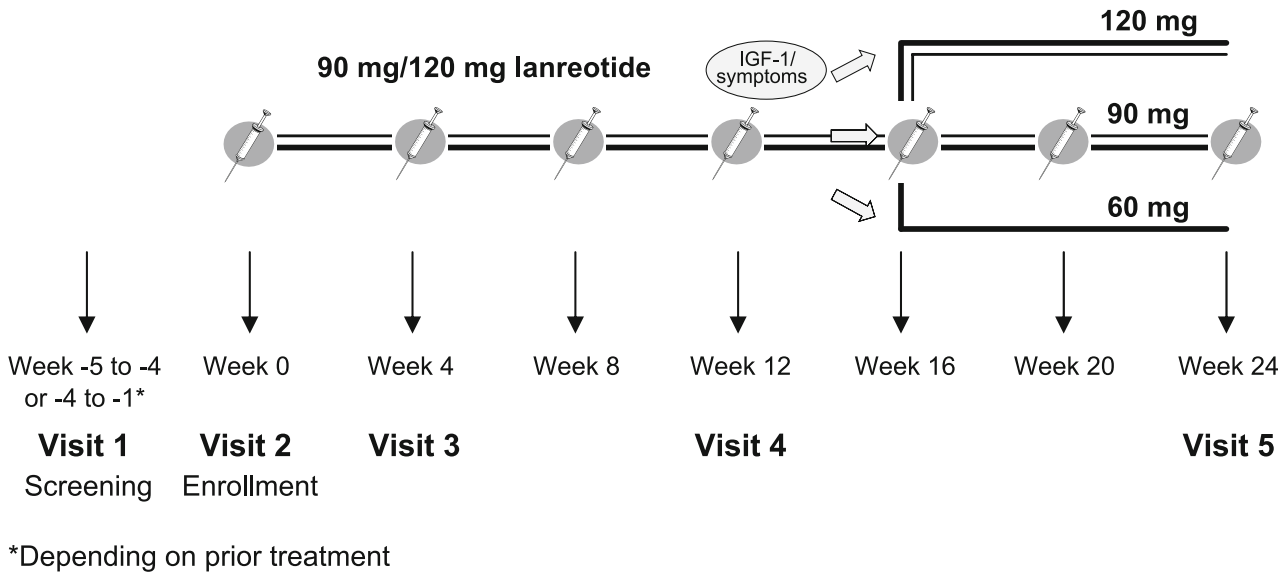


for symptoms was calculated at each visit based on the patient's sweating, snoring, joint pain, headache and fatigue. Each symptom was scored as -2 (always), -1 (most of the time), 0 (sometimes), 1 (rarely) or 2 (never). Switch patients also completed a convenience questionnaire at weeks 0 and 24. All questionnaires were made available in English and Spanish.

The primary endpoint was the percentage of patients/ partners competent to self- or partner-administer lanreotide at study completion as assessed by the health care professional. Secondary assessments included IGF-1 and glucose-suppressed GH levels, symptom and convenience questionnaires and safety assessments. Safety assessments included a physical examination, collection of adverse events (AEs) and laboratory examinations such as a complete blood count (CBC), serum chemistries, thyroid function tests and urinalysis.

\section{Patients}

Fifty-nine patients with acromegaly were recruited by the local investigators and studied at one of thirteen centers in the United States. The study protocol was approved by the local institutional review board of participating centers (see additional details at: www.clinicaltrials.gov; identified: NCT00447499). Informed consent was obtained from the patients and their partners prior to any study-related activities.

The study inclusion criteria included suitably motivated patients with a clinical diagnosis of acromegaly due to a pituitary tumor and age $\geq 18$ years. Switch patients also had to have taken a constant dose of octreotide LAR for at least 3 months, with serum IGF-1 no higher than $10 \%$ above normal. Switch patients had to have their last octreotide LAR injection 28-35 days prior to study enrollment. The study exclusion criteria included pituitary surgery within 3 months of screening, pituitary radiation therapy within 3 years of screening, GH receptor antagonist therapy within 6 months of screening, current octreotide LAR dose $>30 \mathrm{mg}$ every 28 days, renal or hepatic abnormalities, symptomatic cholelithiasis, poorly controlled diabetes or thyroid disease, pregnancy or breastfeeding. Contraception was mandatory in patients of child-bearing potential.

\section{IGF-1 and GH assays}

Serum IGF-1 levels were measured with a radioimmunoassay after acid-alcohol extraction (RIA-AE) via gamma counter (Esoterix; Calabasas Hills, CA). The intra-assay variability of the RIA-AE assay, measured by the coefficient of variation $(\mathrm{CV})$, was $5-14 \%$ based on four samples and five assay runs of each sample. The inter-assay variability of the RIA-AE assay was $7-17 \%$ based on four samples and three assay runs of each sample.

Serum GH levels were measured with an immunochemiluminometric assay (ICMA) using an immunometer (Esoterix; Calabasas Hills, CA). The intra-assay variability of the ICMA assay, measured by the CV, was $4-14 \%$ based on ten samples and four assay runs of each sample. The inter-assay variability of the ICMA assay was $7-14 \%$ based on six samples and seventeen assay runs of each sample.

\section{Statistical analyses}

For the baseline characteristics, frequency counts and percents (\%) were calculated for sex, previous pituitary surgery, prior acromegaly medication, SSA treatment and DA treatment. Mean and standard deviation (SD) were calculated for age, body mass index (BMI), duration of acromegaly, time since last pituitary surgery and time since last SSA treatment. The comparisons between switch and "other" patients were performed using either a chi-square test or Student's $t$-test.

The number and percent of patients who correctly administered the lanreotide injection at week 24 were calculated based on the competency questionnaire completed by the health care professionals for each patient/ partner. For the switch patients, the number and percent of patients who experienced pain (i.e., not painful, somewhat painful, moderately painful and very painful) from the lanreotide injection at week 24 and from the octreotide LAR injection at week 0 were calculated based on the convenience questionnaire completed by the switch patients. The pain experience (not painful) for the two treatments was compared using a McNemar's test. For the preference for future treatment, the number and percent of switch patients who preferred lanreotide or octreotide LAR or who had no preference at week 24 or early termination were calculated based on the convenience questionnaire completed by the switch patients. A chi-square test was used to test the hypothesis that more than $50 \%$ of switch patients would prefer one drug over the other for future use.

The biochemical control of glucose-suppressed GH and IGF-1 were determined at weeks 0 and 24 as: normal IGF-1 and $\mathrm{GH}<1 \mu \mathrm{g} / \mathrm{L}$; normal IGF-1 only; GH $<1 \mu \mathrm{g} / \mathrm{L}$ only or none of the above. For each of these four categories, the number and percent of patients were calculated for switch and "other" patients. A McNemar's test was used to test the change from weeks 0 to 24 within each of the two the groups. No between group comparisons were performed because the switch patients had been taking octreotide LAR for $\geq 3$ months prior to study enrollment and their serum IGF-1 levels were no higher than $10 \%$ above normal. 
Acromegaly symptoms scores were categorized as symptoms present (scores of -2 to 0 ) or absent (scores of 1 or 2), and the total score was used to evaluate symptom control in each patient at weeks 0 and 24. A McNemar's test was used to test the change in symptom status from week 0 to week 24 or early termination within each of the two the groups using the total score.

AEs were coded using the MedDRA dictionary Version 8.1. Possibly and probably treatment-related AEs occurring in more than $5 \%$ of patients were summarized for switch and "other" patients. A chi-square test was used to compare the two groups.

\section{Results}

Thirty-three switch patients (56\% of patients) and 26 "other" patients (44\%) were enrolled in the study between June 2007 and May 2008. The baseline characteristics for all patients are shown in Table 1. As expected, switch patients had a longer duration of acromegaly than "other" patients. Most patients in both groups had undergone pituitary surgery.

Seven patients withdrew early from the study. Three switch patients withdrew early; one patient preferred octreotide LAR, one patient found the interstate travel required to attend study visits to be burdensome and one patient had pituitary tumor progression. Four "other" patients withdrew early from the study; one patient for an adverse event (irritable bowel syndrome), one patient refused the week 24 injection, one investigator withdraw a patient who was suffering from anxiety related to several incidences of abdominal pain, and the sponsor withdraw a patient because of concerns about drug transport and dosing reliability during the patient's overseas travel. All patients were included in the safety analysis.

Twenty-two switch patients started on $90 \mathrm{mg}$ lanreotide and two of these patients were on a DA. Eleven switch patients started on $120 \mathrm{mg}$ lanreotide; ten were switched from $30 \mathrm{mg}$ octreotide LAR and one was switched from $20 \mathrm{mg}$ octreotide LAR. Three of these patients were on a DA. At week 16, three switch patients had their dose adjusted from 90 to $120 \mathrm{mg}$ based on their symptoms and IGF-1 levels at week 12. The rest of the switch patients remained on their original dose for the duration of the study. Twenty-five "other" patients started on $90 \mathrm{mg}$ lanreotide; five of these patients were on a DA. One "other" patient, who was on a DA and had previously been on $30 \mathrm{mg}$ octreotide LAR, started on $120 \mathrm{mg}$ lanreotide. Eleven "other" patients had their dose adjusted from 90 to $120 \mathrm{mg}$ and two had their dose adjusted from 90 to $60 \mathrm{mg}$ based on their symptoms and IGF-1 levels by week 12 . The rest of the "other" patients remained on their original dose for the duration of the study.

\section{Injection competency and tolerability}

Forty-one patients (69.5\%) self-injected lanreotide and 18 partners $(30.5 \%)$ partner-injected lanreotide during the study. Of the 41 patients who chose to self-inject lanreotide, 27 were switch patients $(81.8 \%)$ and 14 were "other" patients $(53.8 \%)$. No patient switched from self-to partnerinjection of lanreotide during the study, although one patient had a partner administer the injection at week 4 and

Table 1 Baseline patient characteristics

\begin{tabular}{lcr}
\hline Characteristic & Switch patients $(n=33)$ & Other patients $(n=26)$ \\
\hline Female, $n(\%)$ & $17(51.5)$ & $13(50.0)$ \\
Age, year, mean (SD) & $54.0(13.0)$ & $51.9(10.5)$ \\
BMI, mean (SD) & $32.0(7.7)$ & $29.7(5.1)$ \\
Duration of acromegaly, year, mean (SD) & $10.4(7.1)$ & $4.3(4.3)^{*}$ \\
Previous pituitary surgery, $n(\%)$ & $30(90.9)$ & $22(84.6)$ \\
Time since last pituitary surgery, year, mean (SD) & $9.1(6.6)$ & $3.4(3.7)^{\dagger}$ \\
Prior acromegaly medication, $n(\%)$ & $33(100.0)$ & $11(42.3)^{*}$ \\
Prior SSA treatment, $n(\%)$ & $33(100.0)$ & $5(19.2)^{*}$ \\
Time since last SSA treatment, days, mean (SD) & $32.0(8.3)$ & $388.0(373.8)^{\mathrm{a}}$ \\
DA treatment, $n(\%)$ & $5(15.2)$ & $6(23.1)$ \\
\hline
\end{tabular}

$B M I$ body mass index, $S S A$ somatostatin analogue, $D A$ dopamine agonist

* $P=0.0003$ versus switch patients

$\dagger P=0.0006$ versus switch patients

* $P<0.0001$ versus switch patients

${ }^{\text {a }}$ Only 5 patients had received prior SSA treatment in this category (short-acting octreotide $[n=1]$ or octreotide LAR $>4$ months prior to study enrollment $[n=4])$ 
self-administered all the other injections. Health care professionals reported that $100 \%$ of patients/partners were able to correctly self- or partner-inject lanreotide by week 4 and continued to correctly administer the injection throughout the study. Health care professionals also reported that they were confident that $100 \%$ of patients/partners were able to follow the instructions given, insert the needle correctly, achieve the appropriate needle depth and administer the complete dose by week 4 .

More switch patients reported that the injection was not painful for lanreotide at week 24 versus octreotide LAR at week $0(50.0 \%$ versus $25.0 \%$ of patients, respectively; $P=0.0201)$. The rest of the switch patients reported the lanreotide and octreotide LAR injections as somewhat painful (43.8 vs. $59.4 \%)$, moderately painful (6.3 vs. $6.3 \%$ ) or very painful $(0.0$ vs. $9.4 \%)$. More switch patients reported that the injection was very convenient for lanreotide at week 24 versus octreotide LAR at week 0 (75.0 vs. $18.8 \%$ of patients, respectively; $P=0.0002$ ). The rest of the switch patients reported the lanreotide and octreotide LAR injections as somewhat convenient (15.6 vs. $18.8 \%$ ), neither convenient nor inconvenient (3.1 vs. 3.1\%), somewhat inconvenient ( 3.1 vs. $43.8 \%$ ) or very inconvenient (3.1 vs. 15.6\%). Most switch patients reported they preferred lanreotide over octreotide LAR for future use (81.3 vs. $12.5 \%$, respectively, $6.2 \%$ had no preference; $P=0.0001)$.

\section{IGF-1 and GH levels}

IGF-1 control was maintained in most switch patients (Fig. 2). Serum IGF-1 levels were normal in 28 switch patients (84.8\%) at week 0 (i.e., while on octreotide LAR) and in 30 switch patients $(93.7 \%)$ at week 24 or early termination. Serum IGF-1 levels were normal in four "other" patients (15.4\%) at week 0 and in 12 "other" patients $(46.2 \%)$ at week 24 or early termination.

Glucose-suppressed GH levels were obtained in most patients; they were not obtained in diabetic patients or all of the early termination patients. Glucose-suppressed GH levels were $<1 \mu \mathrm{g} / \mathrm{L}$ in 21 switch patients $(70.0 \%)$ at week 0 and in 20 switch patients (76.9\%) at week 24 or early termination. Glucose-suppressed GH levels were $<1 \mu \mathrm{g} / \mathrm{L}$ in only three "other" patients $(12.0 \%)$ at week 0 and in 9 "other" patients $(39.1 \%)$ at week 24 or early termination.

Forty-nine patients had both IGF-1 and glucose-suppressed GH values at week 24 or early termination. Self- or partner-administration of lanreotide was associated with both IGF-1 and GH control in many patients with acromegaly $(73.1 \%$ of switch patients and $30.4 \%$ of "other" patients at week 24 or early termination versus $63.3 \%$ and $4.0 \%$ at week 0 , respectively; Table 2).

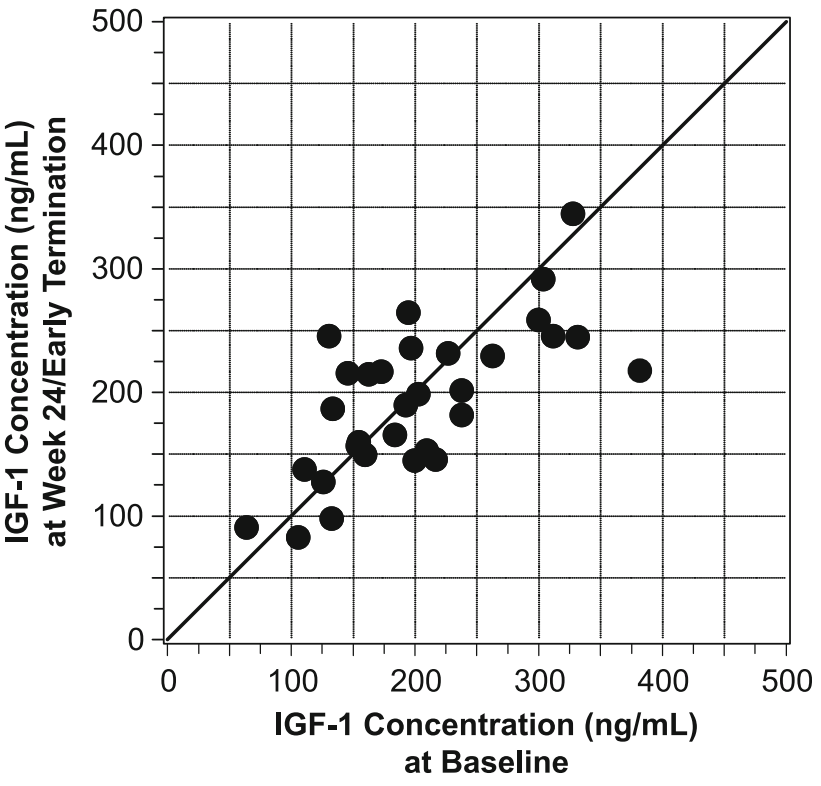

Fig. 2 Serum IGF-1 concentrations at baseline versus week 24 or early termination for switch patients who had these values available at both time points $(n=32)$

Table 2 Normal IGF-1 or glucose-suppressed GH levels at weeks 0 and 24 or at early termination

$\begin{array}{ll}\begin{array}{l}\text { Switch patients } \\ (n=33)\end{array} & \begin{array}{l}\text { Other patients } \\ (n=26)\end{array}\end{array}$

Week $0, n(\%)$

Normal IGF-1 and GH $<1 \mu \mathrm{g} / \mathrm{L} \quad 19 / 30$ (63.3) $\quad 1 / 25$ (4.0)

Normal IGF-1 and GH $>1 \mu \mathrm{g} / \mathrm{L} \quad 6 / 30(20.0) \quad 3 / 25(12.0)$

Elevated IGF-1 and GH $<1 \mu \mathrm{g} / \mathrm{L} \quad 2 / 30$ (6.7) $\quad 2 / 25(8.0)$

Elevated IGF-1 and GH $>1 \mu \mathrm{g} / \mathrm{L} \quad 3 / 30(10.0) \quad 19 / 25$ (76.0)

Week 24 or early termination, $n(\%)$

Normal IGF-1 and GH $<1 \mu \mathrm{g} / \mathrm{L} \quad 19 / 26$ (73.1) $\quad 7 / 23$ (30.4)*

Normal IGF-1 and GH $>1 \mu \mathrm{g} / \mathrm{L} \quad 5 / 26(19.2) \quad 3 / 23(13.0)$

Elevated IGF-1 and GH $<1 \mu \mathrm{g} / \mathrm{L} \quad 1 / 26(3.8) \quad 2 / 23(8.7)$

Elevated IGF-1 and GH $>1 \mu \mathrm{g} / \mathrm{L} \quad 1 / 26(3.8) \quad 11 / 23$ (47.8)

Glucose-suppressed GH levels were not obtained in diabetic patients and were not obtained in all of the early termination patients

$* P=0.0143$ versus week 0

Discordant IGF-1 and glucose-suppressed GH levels $<1 \mu \mathrm{g} / \mathrm{L}$ were observed in 11 of 49 patients $(22.4 \%)$ who had both values available at week 24 or early termination (Table 2). Six switch patients $(23.1 \%)$ had discordant IGF-1 and glucose-suppressed GH levels $<1 \mu \mathrm{g} / \mathrm{L}$ at week 24 or early termination; five of these switch patients (83.3\%) had normal IGF-1 levels only. Five "other" patients $(21.7 \%)$ had discordant IGF-1 and glucosesuppressed GH levels $<1 \mu \mathrm{g} / \mathrm{L}$ at week 24 or early termination; three of these "other" patients $(60.0 \%)$ had normal IGF-1 levels only. Discordant IGF-1 and fasting GH levels 
$<1 \mu \mathrm{g} / \mathrm{L}$ were observed in 18 of 54 patients $(33.3 \%)$ who had both values at week 24 or early termination. Twelve switch (41.4\%) and six "other" patients (24.0\%) had discordant IGF-1 and fasting GH levels $<1 \mu \mathrm{g} / \mathrm{L}$.

\section{Symptoms}

Symptom control was maintained in most switch patients and symptoms improved in many "other" patients from week 0 to 24 or early termination (based on the change in symptom status for the symptoms scores for "other" patients, $P=0.0075$; Fig. 3 ).

\section{Safety}

Self- or partner-administration of lanreotide was generally well tolerated. The most common AE was diarrhea, which was less common among switch than "other" patients. There were no unexpected treatment-related AEs. The possibly/probably treatment-related AEs are shown in Table 3.

There were seven serious AEs in five patients. One serious AE was considered possibly or probably treatmentrelated by the physician: a switch patient with pancreatitis

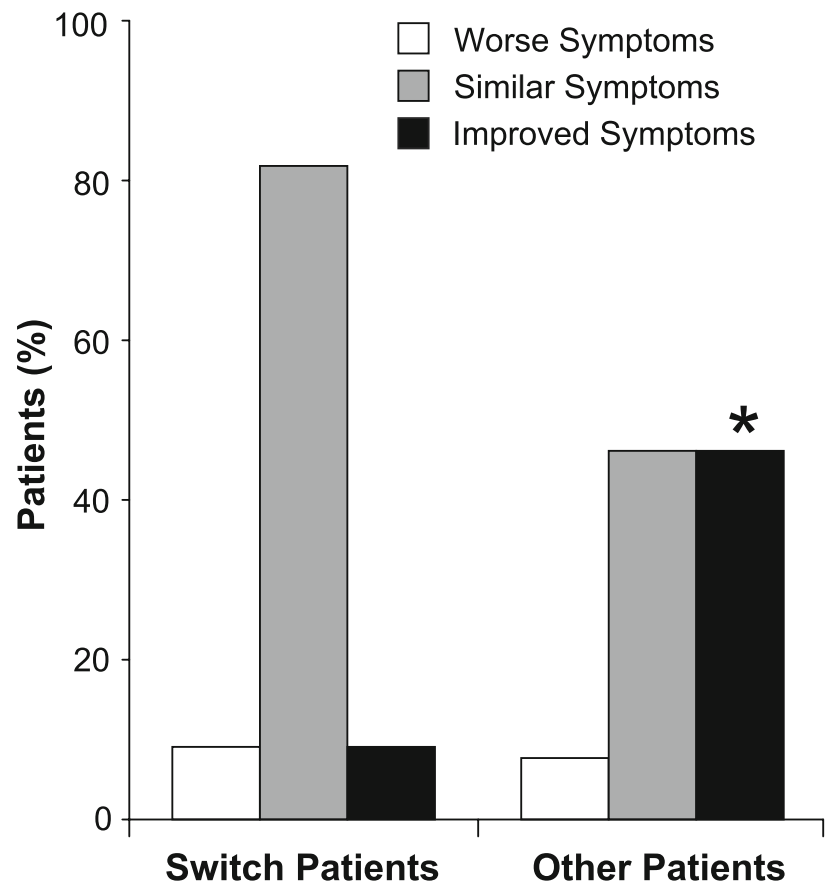

Fig. 3 The percentage of patients with worse, similar or improved acromegaly symptoms from week 0 to 24 or early termination. A total score for symptoms was calculated at each visit based on the patient's sweating, snoring, joint pain, headache and fatigue. Each symptom was scored as -2 (always), -1 (most of the time), 0 (sometimes), 1 (rarely) or 2 (never). ${ }^{*} P=0.0075$ for the change in symptom status for "other" patients from symptoms present (scores of -2 to 0 ) to absent (scores of 1 or 2)
Table 3 Possibly or probably treatment-related AEs (occurring in $>5 \%$ of patients)

\begin{tabular}{lll}
\hline & $\begin{array}{l}\text { Switch } \\
\text { patients } \\
(n=33), \\
n(\%)\end{array}$ & $\begin{array}{l}\text { Other } \\
\text { patients } \\
(n=26), \\
n(\%)\end{array}$ \\
\hline Patients reporting $\geq 1$ adverse event & $18(55)$ & $24(92)^{*}$ \\
Diarrhea & $9(27)$ & $17(65)^{\dagger}$ \\
Headache & $7(21)$ & $3(12)$ \\
Abdominal pain upper & $3(9)$ & $5(19)$ \\
Abdominal pain & $2(6)$ & $6(23)$ \\
Nausea & $4(12)$ & $4(15)$ \\
Injection site pain & $4(12)$ & $2(8)$ \\
Injection site irritation & $2(6)$ & $3(12)$ \\
Alopecia & $1(3)$ & $3(12)$ \\
Flatulence & $2(6)$ & $2(8)$ \\
Injection site pruritus & $1(3)$ & $3(12)$ \\
Serious adverse event: pancreatitis & $1(3)$ & - \\
$\quad$ (resolved) & &
\end{tabular}

$* P=0.0015$ versus switch patients; ${ }^{\dagger} P=0.0034$ versus switch patients

and abdominal pain 1 day after the last lanreotide injection who recovered with no permanent damage. Six serious AEs in four patients were considered unrelated to treatment by the physician: one switch patient developed melanoma; one switch patient had poorly controlled diabetes, worsening obesity and worsening osteoarthritis; one switch patient had worsening chronic back pain and one "other" patient developed thyroid cancer.

\section{Discussion}

Acromegaly is a chronic disease that often requires longterm pharmacological treatment in patients with persistent GH excess after surgery. For many patients, such as those with busy schedules, limited mobility or who live a long distance from the health care professional's office, the possibility of self- or partner-administration of therapy is important as it may decrease the need for frequent trips to the office. For this reason, the use of drug formulations that do not require reconstitution by a health care professional has increased in recent years.

The current study evaluated the efficacy and safety of self- or partner-administration of lanreotide in lanreotidenaïve patients who either switched directly from another SSA (octreotide LAR) or were SSA treatment-naïve or not currently on octreotide LAR. This study found that $100 \%$ of patients/partners were able to correctly self- or partnerinject lanreotide and that no patient reported a preference to receive the injection by a health care professional. 
Accordingly, the majority of patients reported they preferred lanreotide over octreotide LAR for future use. A previous study also found that patients who were on lanreotide for at least 4 months and who received injections by a health care professional were able to successfully switch to self- or partner-administer lanreotide while maintaining GH and IGF-1 control [9].

More patients elected self-injection than partner-injection in this study, which emphasizes the convenience of this approach versus required visits to a health care professional. There was no gender preference for self-administration in this study (69\% of the men and $70 \%$ of the women self-administered lanreotide). Overall, the costs of the two medications are very similar with minor variations depending upon insurance coverage. However, the ability of a patient to self- or partner-inject means that they do not have to travel to a health care facility for injections, with a reduction in transportation, parking, and office cost, and avoidance of lost time from work.

As one might expect, the duration of acromegaly was longer in switch than in "other" patients, since they were previously on a fixed dose of SSA, and their IGF-1 and GH control was maintained with lanreotide treatment in this study. IGF-1 and GH control was lower in the "other" patients because these patients were not pre-selected to be responsive to SSAs. Indeed, the $46.2 \%$ of "other" patients with normalization of serum IGF-1 levels by week 24 or early termination was similar to the efficacy reported previously for lanreotide in patients with acromegaly [10] even with the short duration of follow-up. Consistent with the hormonal data, the symptoms of acromegaly did not change significantly in the switch patients and improved in the "other" patients in this study.

Previous studies have reported discordant serum IGF-1 and GH levels in patients with acromegaly on SSA therapies [11-13]. Discordant values were commonly reported as normal IGF-1 and either high random or post-glucose GH levels, although the reverse has also been reported. A recent study by Carmichael et al. [12] found that glucosesuppressed GH levels $(<2 \mu \mathrm{g} / \mathrm{L}$ when tested before 1998, and $<1 \mu \mathrm{g} / \mathrm{L}$ afterwards) were discordant with serum IGF-1 in $48 \%$ of patients receiving SSAs. The authors concluded that post-glucose GH levels are not useful for the evaluation of disease control in patients receiving SSAs. Another study found discordant serum IGF-1 and glucose-suppressed GH levels in $41 \%$ of patients receiving octreotide LAR [13]. In the current study, discordant IGF-1 and $\mathrm{GH}$ levels at week 24 or at early termination were observed in 11 of 49 patients who had both these values available (22.4\% of patients). Although the reason for the observed difference in discordant IGF-1 and GH levels noted in the current versus previous studies is not clear, one possible explanation may be the use of a single SSA in the current study. Another possible explanation is that the different settings, clinical trial versus retrospective review, may influence the timing of the oral glucose tolerance test (OGTT) relative to the injection, with patients in the current study undergoing OGTT in a more timely fashion relative to the last SSA injection compared with the variable timing typically encountered in clinical practice. The current study, however, was not powered to make this determination. Independent of the explanation for this difference, the data from the current study suggest that post-glucose OGTT might still have a value in assessing disease control in patients with acromegaly on SSAs.

In conclusion, this study found that self- or partneradministration of lanreotide was generally well tolerated and associated with control of IGF-1 and GH levels in many lanreotide-naïve patients with acromegaly. The most common $\mathrm{AE}$ was diarrhea, which was less common among switch than "other" patients. Self- or partner-administration requires instruction by an experienced health care professional, who must supervise the first injection to ensure proper administration. This self-administration approach may give patients the option to decrease their trips to their health care professional.

Acknowledgments This study was sponsored by Tercica, Inc., a subsidiary of the Ipsen Group; the sponsor participated in the study design as well as the data collection, analysis and interpretation. Medical writing support for the preparation of this manuscript was provided by Jessica Mendoza, $\mathrm{PhD}$, who is a full-time employee of Tercica, Inc., a subsidiary of the Ipsen Group.

Open Access This article is distributed under the terms of the Creative Commons Attribution Noncommercial License which permits any noncommercial use, distribution, and reproduction in any medium, provided the original author(s) and source are credited.

\section{Appendix}

The SALSA Study Group consists of the site investigators and coordinators involved in this study and includes: Stephen Aronoff, MD and Lisa Nguyen, CRC (Research Institute of Dallas, Dallas, TX); Vivien Bonert, MD and Stephanie DeLeon, RN, BSN (Cedars-Sinai Medical Center, Los Angeles, CA; David Geffen School of Medicine at University of California, Los Angeles, Los Angeles, CA); David M. Cook, MD and Marie Cook, RN, BSN (Oregon Health \& Science University, Portland, OR); Raymond I. Fink, MD and Denise Humphries, RN (Diabetes \& Endocrine Associates, La Mesa, CA); Pamela U. Freda, MD and Lydia Kotsishevsky, MD (Columbia University, New York, NY); David L. Kleinberg, MD and Sara Lubitz, MD (New York University School of Medicine, New York, NY); Howard A. Lippes, MD and Mary Christine Uebbing, 
NP (R \& B Medical Group, Buffalo, NY); Mark E. Molitch, MD and Daphne Adelman, BSN, MBA (Northwestern University Feinberg School of Medicine, Chicago, IL); Lisa B. Nachtigall, MD and Karen Pulaski-Liebert, RN (Massachusetts General Hospital, Boston, MA); Roberto Salvatori, MD and Margene Kennedy, CRNP (Johns Hopkins University, Baltimore, MD); Morali D. Sharma, MD and Sara Schanzer (Baylor College of Medicine, Houston, TX); Steven G. Waguespack, MD and Cheryl Mize, RN (University of Texas, MD Anderson Cancer Center, Houston, TX); and Margaret E Wierman, MD and Stacey M. Mitchell, BSN, RN (Denver Research Institute, Denver VA Medical Center, Denver, CO).

\section{References}

1. Melmed S (2006) Medical progress: acromegaly. N Engl J Med $355: 2558-2573$

2. Giustina A, Barkan A, Chanson P et al (2008) Guidelines for the treatment of growth hormone excess and growth hormone deficiency in adults. J Endocrinol Invest 31:820-838

3. Shih HA, Loeffler JS (2008) Radiation therapy in acromegaly. Rev Endocr Metab Disord 9:59-65

4. Newman CB, Melmed S, George A et al (1998) Octreotide as primary therapy for acromegaly. J Clin Endocrinol Metab 83:3034-3040

5. Carlsen SM, Lund-Johansen M, Schreiner T et al (2008) Preoperative octreotide treatment in newly diagnosed acromegalic patients with macroadenomas increases cure short-term postoperative rates: a prospective, randomized trial. J Clin Endocrinol Metab 93:2984-2990

6. Melmed S, Casanueva F, Cavagnini F et al (2005) Consensus statement: medical management of acromegaly. Eur J Endocrinol 153:737-740

7. Melmed S, Sternberg R, Cook D et al (2005) A critical analysis of pituitary tumor shrinkage during primary medical therapy in acromegaly. J Clin Endocrinol Metab 90:4405-4410

8. Bronstein M, Musolino N, Jallad R et al (2005) Pharmacokinetic profile of lanreotide Autogel in patients with acromegaly after four deep subcutaneous injections of 60,90 or $120 \mathrm{mg}$ every 28 days. Clin Endocrinol (Oxf) 63:514-519

9. Bevan JS, Newell-Price J, Wass JA et al (2008) Home administration of lanreotide Autogel by patients with acromegaly, or their partners, is safe and effective. Clin Endocrinol (Oxf) 68:343-349

10. Caron P, Beckers A, Cullen DR et al (2002) Efficacy of the new long-acting formulation of lanreotide (lanreotide autogel) in the management of acromegaly. J Clin Endocrinol Metab 87:99-104

11. Machado EO, Taboada GF, Neto LV et al (2008) Prevalence of discordant GH and IGF-I levels in acromegalics at diagnosis, after surgical treatment and during treatment with octreotide LAR. Growth Horm IGF Res 18:389-393

12. Carmichael JD, Bonert VS, Mirocha JM, Melmed S (2009) The utility of oral glucose tolerance testing for diagnosis and assessment of treatment outcomes in 166 patients with acromegaly. J Clin Endocrinol Metab 94:523-527

13. Yetkin DO, Boysan SN, Tiryakioglu O, Yalin AS, Kadioglu P (2007) Forty month follow-up of persistent and difficultly controlled acromegalic patients treated with depot long acting somatostatin analog octreotide. Endocr J 54:459-464 